\title{
Analysis of a Unilateral Contact Problem with Normal Compliance
}

\author{
Arezki Touzaline and Rachid Guettaf
}

\begin{abstract}
The paper deals with the study of a quasistatic unilateral contact problem between a nonlinear elastic body and a foundation. The contact is modelled with a normal compliance condition associated to unilateral constraint and the Coulomb's friction law. The adhesion between contact surfaces is taken into account and is modelled with a surface variable, the bonding field, whose evolution is described by a first-order differential equation. We establish a variational formulation of the mechanical problem and prove an existence and uniqueness result in the case where the coefficent of friction is bounded by a certain constant. The technique of the proof is based on arguments of time-dependent variational inequalities, differential equations and fixed-point theorem.
\end{abstract}

AMS Subject Classification (2000). 74M10, 74M15, 49J40.

Keywords. elastic, normal compliance, adhesion,friction, unilateral, weak solution.

\section{Introduction}

Contact problems involving deformable bodies are quite frequent in industry as well as in daily life and play an important role in structural and mechanical systems. Contact processes involve complicated surface phenomena, and 
are modelled with highly nonlinear initial boundary value problems. Taking into account various contact conditions associated with more and more complex behavior laws lead to the introduction of new and non standard models, expressed by the aid of evolution variational inequalities. An early attempt to study contact problems within the framework of variational inequalities was made in [11]. The mathematical, mechanical and numerical state of the art can be found in $[2,19,33]$. Unilateral frictional contact problems involving Signorini's condition with or without adhesion were studied by several authors, see for instance the references in $[1,3,6,7,9,10,12,18,24,34,35]$. Recently new and nonstandard contact condition which includes memory effects is used, see for instance [20,21]. The new articles also appeared which analyze the piezoelectricity, see $[31,32]$.

In this paper, we study a mathematical model which describes a frictional unilateral contact problem with adhesion between a nonlinear elastic body and a deformable foundation. Following $[17,34]$ the contact is modelled with a normal compliance condition associated to unilateral constraint, where the penetration is limited. Recall that models for dynamic or quasistatic processes of frictionless adhesive contact between a deformable body and a foundation have been studied in $[4,5,13,18,23,27,28,30,34]$. Also recently dynamic or quasistatic frictional contact problems with adhesion were studied in $[7,8,9,23,25,35]$. Here as in $[14,15]$ we use the bonding field as an additional state variable $\beta$, defined on the contact surface of the boundary. The variable is restricted to values $0 \leq \beta \leq 1$; when $\beta=0$ all the bonds are severed and there are no active bonds, when $\beta=1$ all the bonds are active; when $0<\beta<1$ it measures the fraction of active bonds and partial adhesion takes place. We refer the reader to the extensive bibliography on the subject in $[3,13,14,16,23,26,27]$.

The novelty of this work is that we extend the result established in [35] by generalizing the assumption on the adhesive constraints introduced in $[27,35]$ to arbitrary adhesive constraints. We establish a variational formulation of the mechanical problem for which we prove the existence of a unique weak solution if the coefficient of friction satisfies a certain condition and obtain a partial regularity result for the solution.

The paper is structured as follows. In section 2 we present some notations and preliminaries. In section 3 we state the mechanical model of elastic frictional contact with adhesion and give a variational formulation. In section 4 we state and prove our main existence and uniqueness result, Theorem 4.1. 


\section{Notations and preliminaries}

We denote by $S_{d}$ the space of second order symmetric tensors on $\mathbb{R}^{d}(d=2,3)$ and |.| represents the Euclidean norm on $\mathbb{R}^{d}$ and $S_{d}$. Thus, for every $u, v \in$ $\mathbb{R}^{d}, u . v=u_{i} v_{i},|v|=(v \cdot v)^{\frac{1}{2}}$, and for every $\sigma, \tau \in S_{d}, \sigma \cdot \tau=\sigma_{i j} \tau_{i j},|\tau|=$ $(\tau . \tau)^{\frac{1}{2}}$. Here and below, the indices $i$ and $j$ run between 1 and $d$ and the summation convention over repeated indices is adopted. We shall use the notation:

$$
\begin{aligned}
& H=\left(L^{2}(\Omega)\right)^{d}, H_{1}=\left(H^{1}(\Omega)\right)^{d}, Q=\left\{\tau=\left(\tau_{i j}\right) ; \tau_{i j}=\tau_{j i} \in L^{2}(\Omega)\right\}, \\
& Q_{1}=\{\tau \in Q ; \operatorname{div} \tau \in H\} .
\end{aligned}
$$

Note that $H$ and $Q$ are real Hilbert spaces endowed with the respective canonical inner products

$$
(u, v)_{H}=\int_{\Omega} u_{i} v_{i} d x, \quad(\sigma, \tau)_{Q}=\int_{\Omega} \sigma_{i j} \tau_{i j} d x .
$$

The strain tensor is

$$
\varepsilon(u)=\left(\varepsilon_{i j}(u)\right)=\frac{1}{2}\left(u_{i, j}+u_{j, i}\right) ;
$$

$\operatorname{div} \sigma=\left(\sigma_{i j, j}\right)$ is the divergence of $\sigma$. For every $v \in H_{1}$ we denote by $v_{\nu}$ and $v_{\tau}$ the normal and tangential components of $v$ on the boundary $\Gamma$ given by

$$
v_{\nu}=v . \nu, \quad v_{\tau}=v-v_{\nu} \nu .
$$

We also denote by $\sigma_{\nu}$ and $\sigma_{\tau}$ the normal and the tangential traces of a function $\sigma \in Q_{1}$, and when $\sigma$ is a regular function then

$$
\sigma_{\nu}=(\sigma \nu) . \nu, \quad \sigma_{\tau}=\sigma \nu-\sigma_{\nu} \nu
$$

and the following Green's formula holds:

$$
(\sigma, \varepsilon(v))_{Q}+(\operatorname{div} \sigma, v)_{H}=\int_{\Gamma} \sigma \nu \cdot v d a \quad \forall v \in H_{1},
$$

where $d a$ is the surface measure element. For $p \in[1, \infty]$, we use the standard norm of $L^{p}(0, T ; V)$. We also use the Sobolev space $W^{1, \infty}(0, T ; V)$ equipped with the norm

$$
\|v\|_{W^{1, \infty}(0, T ; V)}=\|v\|_{L^{\infty}(0, T ; V)}+\|\dot{v}\|_{L^{\infty}(0, T ; V)} .
$$

For every real Banach space $\left(X,\|\cdot\|_{X}\right)$ and $T>0$ we use the notation $C([0, T] ; X)$ for the space of continuous functions from $[0, T]$ to $X$; recall that $C([0, T] ; X)$ is a real Banach space with the norm

$$
\|x\|_{C([0, T] ; X)}=\max _{t \in[0, T]}\|x(t)\|_{X} .
$$




\section{Problem statement and variational formulation}

We consider a nonlinear elastic body which occupies a domain $\Omega \subset \mathbb{R}^{d}$ $(d=2,3)$ and assume that its boundary $\Gamma$ is regular and partitioned into three measurable and disjoint parts $\Gamma_{1}, \Gamma_{2}, \Gamma_{3}$ such that meas $\left(\Gamma_{1}\right)>0$. The body is acted upon by a volume force of density $\varphi_{1}$ on $\Omega$ and a surface traction of density $\varphi_{2}$ on $\Gamma_{2}$. On $\Gamma_{3}$ the body is in unilateral and adhesive contact following the Coulomb's friction law with a deformable foundation. Thus, the classical formulation of the mechanical problem is written as follows.

Problem $P_{1}$. Find a displacement $u: \Omega \times[0, T] \rightarrow \mathbb{R}^{d}$ and a bonding field $\beta: \Gamma_{3} \times[0, T] \rightarrow \mathbb{R}$ such that

$$
\begin{aligned}
& \operatorname{div} \sigma+\varphi_{1}=0 \text { in } \Omega \times(0, T), \\
& \sigma=F \varepsilon(u) \text { in } \Omega \times(0, T), \\
& u=0 \quad \text { on } \Gamma_{1} \times(0, T), \\
& \sigma \nu=\varphi_{2} \quad \text { on } \Gamma_{2} \times(0, T), \\
& \left.\begin{array}{l}
u_{\nu} \leq g, \sigma_{\nu}+p\left(u_{\nu}\right)+p_{\nu}\left(\beta, u_{\nu}\right) \leq 0 \\
\left(\sigma_{\nu}+p\left(u_{\nu}\right)+p_{\nu}\left(\beta, u_{\nu}\right)\right)\left(u_{\nu}-g\right)=0
\end{array}\right\} \text { on } \Gamma_{3} \times(0, T) \text {, } \\
& \left.\begin{array}{c}
\left|\sigma_{\tau}+p_{\tau}\left(\beta, u_{\tau}\right)\right| \leq \mu p\left(u_{\nu}\right) \\
\left|\sigma_{\tau}+p_{\tau}\left(\beta, u_{\tau}\right)\right|<\mu p\left(u_{\nu}\right) \Longrightarrow u_{\tau}=0 \\
\left|\sigma_{\tau}+p_{\tau}\left(\beta, u_{\tau}\right)\right|=\mu p\left(u_{\nu}\right) \Longrightarrow \\
\exists \lambda \geq 0 ; \mu p\left(u_{\nu}\right)=-\left(\sigma_{\tau}+p_{\tau}\left(\beta, u_{\tau}\right)\right)
\end{array}\right\} \text { on } \Gamma_{3} \times(0, T) \\
& \dot{\beta}=H_{a d}\left(\beta, R\left(\left|u_{\tau}\right|\right)\right) \text { on } \Gamma_{3} \times(0, T), \\
& \beta(0)=\beta_{0} \text { on } \Gamma_{3} \text {. }
\end{aligned}
$$

Equation (2.1) represents the equilibrium equation. Equation (2.2) represents the elastic constitutive law of the material in which $F$ is a given function and $\varepsilon(u)$ denotes the strain tensor; (2.3) and (2.4) are the displacement and traction boundary conditions, respectively, in which $\nu$ denotes the unit outward normal vector on $\Gamma$ and $\sigma \nu$ represents the Cauchy stress vector. The condition (2.5) represents the unilateral contact with adhesion in which $p$ and $p_{\nu}$ are the normal contact functions. A usual choice of the function $p_{\nu}$ is (see $[20]$ )

$$
p_{\nu}\left(\beta, u_{\nu}\right)=-c_{\nu} \beta^{2} R_{\nu}\left(u_{\nu}\right)
$$


where $c_{\nu}$ is a given adhesion coefficient and $R_{\nu}$ is a truncation operator defined by

$$
R_{\nu}(s)=\left\{\begin{array}{l}
L \text { if } s<-L \\
-s \text { if }-L \leq s \leq 0 \\
0 \text { if } s>0
\end{array} .\right.
$$

Here $L>0$ is the characteristic length of the bond, beyond which the latter has no additional traction (see [27]) and $p$ is a normal compliance function which satisfies the assumption below (3.7). We denote by the positive constant $g$ the maximum value of the penetration. When $u_{\nu}<0$ i.e. when there is separation between the body and the foundation then the condition (2.5) combined with assumption (3.7) on the function $p$ shows that $\sigma_{\nu}=-p_{\nu}\left(\beta, u_{\nu}\right)$ and by assumption (3.8) below, it does not exeed the value $L_{\nu}(1+g)$. When $g>0$, the body may interpenetrate into the foundation, but the penetration is limited that is $u_{\nu} \leq g$. In this case of penetration (i.e. $\left.u_{\nu} \geq 0\right)$, when $0 \leq u_{\nu}<g$ then $-\sigma_{\nu}=p\left(u_{\nu}\right)$ which means that the reaction of the foundation is uniquely determined by the normal displacement and $\sigma_{\nu} \leq 0$. Since $p$ is an increasing function then the reaction of the foundation is increasing with the penetration and when $u_{\nu}=g$, then $-\sigma_{\nu} \geq p(g)$ and $\sigma_{\nu}$ is not uniquely determined. When $g>0$ and $p=0$, condition (2.5) becomes the Signorini contact condition with adhesion with a gap function,

$$
u_{\nu} \leq g, \sigma_{\nu}+p_{\nu}\left(\beta, u_{\nu}\right) \leq 0,\left(\sigma_{\nu}+p_{\nu}\left(\beta, u_{\nu}\right)\right)\left(u_{\nu}-g\right)=0 .
$$

When $g=0$, the condition (2.5) combined with assumption (3.7) becomes the Signorini contact condition with adhesion with a zero gap function, given by

$$
u_{\nu} \leq 0, \sigma_{\nu}+p_{\nu}\left(\beta, u_{\nu}\right) \leq 0,\left(\sigma_{\nu}+p_{\nu}\left(\beta, u_{\nu}\right)\right) u_{\nu}=0 .
$$

This contact condition was used in $[17,18,28,33,34]$. The condition (2.6) represents the frictional contact in which $p_{\tau}$ is an adhesive traction (see [23]) and also a usual choice is

$$
p_{\tau}\left(\beta, u_{\tau}\right)=c_{\tau} \beta^{2} R_{\tau}\left(u_{\tau}\right),
$$

where $c_{\tau}$ is a coefficient of adhesion and $R_{\tau}$ is a truncation operator defined by

$$
R_{\tau}(v)=\left\{\begin{array}{l}
v \text { if }|v| \leq L \\
L \frac{v}{|v|} \text { if }|v|>L
\end{array},\right.
$$

where $L>0$ is the characteristic length of the bonds. We use it in $H_{a d}$, since usually when the glue is stretched beyond the limit $L$, it does not contribue more to the bond strength. Examples of such functions consider

$$
H_{a d}(\beta, r)=-\gamma_{\nu} \beta_{+} r^{2}
$$


or

$$
H_{a d}(\beta, r)=-\gamma_{\nu} \frac{\beta_{+} r^{2}}{1+\beta_{+}}
$$

where $\gamma_{\nu}$ is an adhesion coefficient and $\beta_{+}=\max (0, \beta) ; \lambda$ is a positive parameter which means that the tangential constraint $\left(\sigma_{\tau}+p_{\tau}\left(\beta, u_{\tau}\right)\right)$ opposes the tangential displacement $u_{\tau}$. Equation (2.7) represents the ordinary differential equation which describes the evolution of the bonding field, where $H_{a}$ (see [30]) is a general function discussed below, which vanishes when its first argument vanishes. The function $R: \mathbb{R}_{+} \rightarrow \mathbb{R}_{+}$is a truncation operator defined by

$$
R(s)=\left\{\begin{array}{l}
s \text { if } 0<s<L \\
L \text { if } s \geq L
\end{array}\right.
$$

Since $\dot{\beta} \leq 0$ on $\Gamma_{3} \times(0, T)$, once debonding occurs bonding cannot be reestablished and, indeed, the adhesive process is irreversible. Also from [22] it must be pointed out clearly that condition (2.7) does not allow for complete debonding in finite time. Finally, (2.8) is the initial condition, in which $\beta_{0}$ denotes the initial bonding field. In (2.7) a dot above a variable represents its derivative with respect to time.

We turn now to the variational formulation of Problem $P_{1}$. We denote by $V$ be the closed subspace of $H_{1}$ defined by

$$
V=\left\{v \in H_{1}: v=0 \text { on } \Gamma_{1}\right\}
$$

and let the convex subset of admissible displacements given by

$$
K=\left\{v \in V: v_{\nu} \leq g \text { a.e. on } \Gamma_{3}\right\} .
$$

Since meas $\left(\Gamma_{1}\right)>0$, the following Korn's inequality holds [11],

$$
\|\varepsilon(v)\|_{Q} \geq c_{\Omega}\|v\|_{H_{1}} \quad \forall v \in V
$$

where $c_{\Omega}>0$ is a constant which depends only on $\Omega$ and $\Gamma_{1}$. We equip $V$ with the inner product

$$
(u, v)_{V}=(\varepsilon(u), \varepsilon(v))_{Q}
$$

and $\|.\|_{V}$ is the associated norm. It follows from Korn's inequality (3.1) that the norms $\|\cdot\|_{H_{1}}$ and $\|\cdot\|_{V}$ are equivalent on $V$. Then $\left(V,\|\cdot\|_{V}\right)$ is a real Hilbert space. Moreover by Sobolev's trace theorem, there exists $d_{\Omega}>0$ which only depends on the domain $\Omega, \Gamma_{1}$ and $\Gamma_{3}$ such that

$$
\|v\|_{\left(L^{2}\left(\Gamma_{3}\right)\right)^{d}} \leq d_{\Omega}\|v\|_{V} \quad \forall v \in V
$$


We suppose that the body forces and surface tractions have the regularity

$$
\varphi_{1} \in W^{1, \infty}(0, T ; H), \quad \varphi_{2} \in W^{1, \infty}\left(0, T ;\left(L^{2}\left(\Gamma_{2}\right)\right)^{d}\right)
$$

and denote by $f(t)$ the element of $V$ defined by

$$
(f(t), v)_{V}=\int_{\Omega} \varphi_{1}(t) \cdot v d x+\int_{\Gamma_{2}} \varphi_{2}(t) . v d a \quad \forall v \in V, t \in[0, T] .
$$

Using (3.3) and (3.4) yields

$$
f \in W^{1, \infty}(0, T ; V) .
$$

In the study of the mechanical problem $P_{1}$ we assume that the nonlinear elasticity operator $F: \Omega \times S_{d} \rightarrow S_{d}$ satisfies:

(a) There exists $M>0$ such that $\left|F\left(x, \varepsilon_{1}\right)-F\left(x, \varepsilon_{2}\right)\right| \leq M\left|\varepsilon_{1}-\varepsilon_{2}\right| \quad \forall \varepsilon_{1}, \varepsilon_{2} \in S_{d}$, a.e. $x \in \Omega$;

(b) there exists $m>0$ such that

$$
\begin{aligned}
& \left(F\left(x, \varepsilon_{1}\right)-F\left(x, \varepsilon_{2}\right)\right) .\left(\varepsilon_{1}-\varepsilon_{2}\right) \geq m\left|\varepsilon_{1}-\varepsilon_{2}\right|^{2}, \\
& \forall \varepsilon_{1}, \varepsilon_{2} \in S_{d}, \text { a.e. } x \in \Omega ;
\end{aligned}
$$

(c) the mapping $x \rightarrow F(x, \varepsilon)$ is Lebesgue measurable on $\Omega$,

for any $\varepsilon \in S_{d}$;

(d) $F(x, 0)=0$ for a.e. $x \in \Omega$.

The coefficient of friction $\mu$ satisfies

$$
\mu \in L^{\infty}\left(\Gamma_{3}\right) \text { and } \mu \geq 0 \text { a.e. on } \Gamma_{3} \text {. }
$$

Next we define the functional $\phi: L^{2}\left(\Gamma_{3}\right) \times V \times V \rightarrow \mathbb{R}$ by

$$
\begin{aligned}
& \phi(\beta, u, v)=\int_{\Gamma_{3}}\left[\left(p\left(u_{\nu}\right)+p_{\nu}\left(\beta, u_{\nu}\right)\right) v_{\nu}+p_{\tau}\left(\beta, u_{\tau}\right) . v_{\tau}\right] d a, \\
& \forall(\beta, u, v) \in L^{2}\left(\Gamma_{3}\right) \times V \times V
\end{aligned}
$$

and the functional $j: V \times V \rightarrow \mathbb{R}_{+}$by

$$
j(u, v)=\int_{\Gamma_{3}} \mu p\left(u_{\nu}\right)\left|v_{\tau}\right| d a \quad \forall(u, v) \in V \times V .
$$

We assume that the normal compliance function $p: \Gamma_{3} \times \mathbb{R} \rightarrow \mathbb{R}_{+}$satisfies:

( (a) There exists $L_{p}>0$ such that

$$
\begin{aligned}
& \left|p\left(x, r_{1}\right)-p\left(x, r_{2}\right)\right| \leq L_{p}\left|r_{1}-r_{2}\right| \\
& \forall r_{1}, r_{2} \in \mathbb{R} \text {, a.e. } x \in \Gamma_{3} ;
\end{aligned}
$$

(b) $\left(p\left(x, r_{1}\right)-p\left(x, r_{2}\right)\right)\left(r_{1}-r_{2}\right) \geq 0$ $\forall r_{1}, r_{2} \in \mathbb{R}$, a.e. $x \in \Gamma_{3}$;

(c) the mapping $x \rightarrow p(x, r)$ is Lebesgue measurable on $\Gamma_{3}$, for any $r \in \mathbb{R}$;

(d) $p(x, r)=0 \forall r \leq 0$, a.e. $x \in \Gamma_{3}$. 
The adhesive normal function $p_{\nu}: \Gamma_{3} \times \mathbb{R} \times \mathbb{R} \rightarrow \mathbb{R}$ satisfies:

(a) There exists $L_{\nu}>0$ such that

$\left|p_{\nu}\left(x, \beta_{1}, r_{1}\right)-p_{\nu}\left(x, \beta_{2}, r_{2}\right)\right| \leq L_{\nu}\left(\left|\beta_{1}-\beta_{2}\right|+\left|r_{1}-r_{2}\right|\right)$

$\forall \beta_{1}, \beta_{2} \in \mathbb{R}, r_{1}, r_{2} \in \mathbb{R}$, a.e. $x \in \Gamma_{3} ;$

(b) $\left(p_{\nu}\left(x, \beta, r_{1}\right)-p_{\nu}\left(x, \beta, r_{2}\right)\right)\left(r_{1}-r_{2}\right) \geq 0$

$\forall \beta_{1}, \beta_{2} \in \mathbb{R}, r_{1}, r_{2} \in \mathbb{R}$, a.e. $x \in \Gamma_{3}$;

(c) the mapping $x \rightarrow p_{\nu}(x, \beta, r)$ is measurable on $\Gamma_{3}$, for any $\beta, r \in \mathbb{R}$;

(d) $p_{\nu}(x, \beta, r)=0 \forall r \geq 0, \beta \in \mathbb{R}$, a.e. $x \in \Gamma_{3}$;

(e) $p_{\nu}(x, 0, r)=0 \forall r \in \mathbb{R}$, a.e. $x \in \Gamma_{3}$.

The adhesive tangential function $p_{\tau}: \Gamma_{3} \times \mathbb{R}^{d} \times \mathbb{R}^{d} \rightarrow \mathbb{R}$ satisfies:

(a) There exists $L_{\tau}>0$ such that

$\left|p_{\tau}\left(x, \beta_{1}, r_{1}\right)-p_{\tau}\left(x, \beta_{2}, r_{2}\right)\right| \leq L_{\tau}\left(\left|\beta_{1}-\beta_{2}\right|+\left|r_{1}-r_{2}\right|\right)$

$\forall \beta_{1}, \beta_{2} \in \mathbb{R}, r_{1}, r_{2} \in \mathbb{R}^{d}$, a.e. $x \in \Gamma_{3}$;

(b) $\left(p_{\tau}\left(x, \beta, r_{1}\right)-p_{\tau}\left(x, \beta, r_{2}\right)\right) \cdot\left(r_{1}-r_{2}\right) \geq 0$

$\forall \beta_{1}, \beta_{2} \in \mathbb{R}, r_{1}, r_{2} \in \mathbb{R}^{d}$, a.e. $x \in \Gamma_{3} ;$

(c) the map $x \rightarrow p_{\tau}(x, \beta, r)$ is measurable on $\Gamma_{3}$, for any $\beta \in \mathbb{R}, r \in \mathbb{R}^{d}$;

(d) the map $x \rightarrow p_{\tau}(x, 0,0) \in\left(L^{\infty}\left(\Gamma_{3}\right)\right)^{d}$,

(e) $p_{\tau}(x, \beta, r) \cdot \nu(x)=0$ for all $r \in \mathbb{R}^{d}$, such that $r . \nu(x)=0$, a.e. $x \in \Gamma_{3}$.

The adhesive rate function $H_{a d}: \Gamma_{3} \times \mathbb{R} \times \mathbb{R} \times[-L, L] \rightarrow \mathbb{R}$ satisfies:

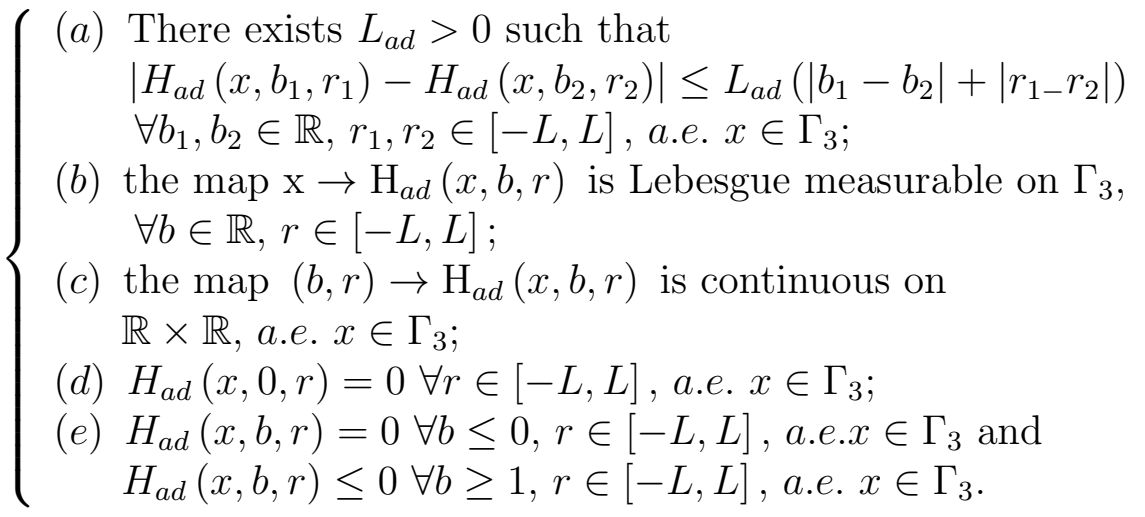

We also assume that the initial bonding field satisfies:

$$
\beta_{0} \in L^{2}\left(\Gamma_{3}\right) ; 0 \leq \beta_{0} \leq 1 \text { a.e. on } \Gamma_{3}
$$

and we need to introduce the set:

$$
B=\left\{\theta:[0, T] \rightarrow L^{2}\left(\Gamma_{3}\right) ; 0 \leq \theta(t) \leq 1, \forall t \in[0, T] \text {, a.e. on } \Gamma_{3}\right\} .
$$


Finally, assuming the solution to be sufficiently regular and applying Green's formula, we deduce the following variational formulation of the mechanical problem $P_{1}$.

Problem $P_{2}$. Find a displacement field $u:[0, T] \rightarrow V$ and a bonding field $\beta:[0, T] \rightarrow L^{2}\left(\Gamma_{3}\right)$ such that

$$
\begin{gathered}
u(t) \in K,(F \varepsilon(u(t)), \varepsilon(v)-\varepsilon(u(t)))_{Q}+\phi(\beta(t), u(t), v-u(t)) \\
+j(u(t), v)-j(u(t), u(t)) \geq(f(t), v-u(t))_{V} \quad \forall v \in K, t \in[0, T] \\
\dot{\beta}(t)=H_{a d}\left(\beta(t), R\left(\left|u_{\tau}(t)\right|\right)\right) \quad \text { a.e. } t \in(0, T), \\
\beta(0)=\beta_{0} .
\end{gathered}
$$

\section{Existence and uniqueness result}

Our main result which will be established in this section is the following theorem.

Theorem 4.1. Let $(3.3),(3.5)-(3.11)$ hold. Then Problem $P_{2}$ has a unique solution, which satisfies

$$
\begin{gathered}
u \in W^{1, \infty}(0, T ; V) \cap C([0, T] ; K) \text { and } \\
\beta \in W^{1, \infty}\left(0, T ; L^{2}\left(\Gamma_{3}\right)\right) \cap B,
\end{gathered}
$$

if

$$
\|\mu\|_{L^{\infty}\left(\Gamma_{3}\right)}<m / L_{p} d_{\Omega}^{2}
$$

The proof of Theorem 4.1 is carried out in several steps. In the first step, let $k>0$ and consider the closed subset $X$ of $C\left([0, T] ; L^{2}\left(\Gamma_{3}\right)\right)$ defined as

$$
X=\left\{\theta \in C\left([0, T] ; L^{2}\left(\Gamma_{3}\right)\right) \cap B, \theta(0)=\beta_{0}\right\},
$$

where the Banach space $C\left([0, T] ; L^{2}\left(\Gamma_{3}\right)\right)$ is endowed with the norm

$$
\|\theta\|_{X}=\max _{t \in[0, T]}\left[\exp (-k t)\|\theta(t)\|_{L^{2}\left(\Gamma_{3}\right)}\right] \text { for all } \theta \in C\left([0, T] ; L^{2}\left(\Gamma_{3}\right)\right) .
$$

Next for a given $\beta \in X$, we consider the following variational problem.

Problem $P_{1 \beta}$. Find $u_{\beta}:[0, T] \rightarrow V$ such that

$$
\begin{aligned}
& u_{\beta}(t) \in K, \quad\left(F \varepsilon\left(u_{\beta}(t)\right), \varepsilon\left(v-u_{\beta}(t)\right)\right)_{Q}+\phi\left(\beta(t), u_{\beta}(t), v-u_{\beta}(t)\right) \\
& +j\left(u_{\beta}(t), v\right)-j\left(u_{\beta}(t), u_{\beta}(t)\right) \geq\left(f(t), v-u_{\beta}(t)\right)_{V} \quad \forall v \in K, t \in[0, T] .
\end{aligned}
$$


We have the following result.

Proposition 4.2. Problem $P_{1 \beta}$ has a unique solution

$$
u_{\beta} \in C([0, T] ; K),
$$

if

$$
\|\mu\|_{L^{\infty}\left(\Gamma_{3}\right)}<m / L_{p} d_{\Omega}^{2} .
$$

The proof of Proposition 4.2 can be established in several steps. Indeed, in the first step for each $t \in[0, T]$ and a given $\eta \in K$, we consider the following intermediate problem.

Problem $P_{\beta \eta}$. Find $u_{\beta \eta}(t) \in K$ such that

$$
\begin{aligned}
& \left(F \varepsilon\left(u_{\beta \eta}(t)\right), \varepsilon\left(v-u_{\beta \eta}(t)\right)\right)_{Q}+\phi\left(\beta(t), u_{\beta \eta}(t), v-u_{\beta \eta}(t)\right)+j(\eta, v) \\
& -j\left(\eta, u_{\beta \eta}(t)\right) \geq\left(f(t), v-u_{\beta \eta}(t)\right)_{V} \quad \forall v \in K .
\end{aligned}
$$

Lemma 4.3. Problem $P_{\beta \eta}$ has a unique solution.

Proof. Let the operator $A_{\beta(t)}: V \rightarrow V$ defined by

$$
\left(A_{\beta(t)} u, v\right)_{V}=(F \varepsilon(u), \varepsilon(v))_{Q}+\phi(\beta(t), u, v), \forall u, v \in V .
$$

We use (3.2), (3.5) (a), (3.5) (b), (3.7) (a), (3.7) (b), (3.8) (a), (3.8) (b), (3.9) (a) and $(3.9)(b)$ to show that the operator $A_{\beta(t)}$ is strongly monotone and Lipschitz continuous; the functional $j(\eta,):. K \rightarrow \mathbb{R}_{+}$is convex and lower semicontinuous; then by a standard existence and uniqueness result for elliptic variational inequalities (see [29]), it follows that there exists a unique element $u_{\beta \eta}(t) \in K$ which satisfies the inequality (4.5) since $K$ is a non-empty, closed convex subset of $V$

Now, in the second step, for a fixed $t \in[0, T]$ we consider the map $T_{t}: K \rightarrow K$ defined as

$$
T_{t}(\eta)=u_{\beta \eta}(t) .
$$

We have the following lemma.

Lemma 4.4. The map $T_{t}$ has a unique fixed point $\eta^{*}$ and $u_{\beta \eta^{*}}(t)$ is a unique solution of the inequality (4.3).

Proof. Let $\eta_{1}, \eta_{2} \in K$. In inequality (4.5) satisfied by $u_{\eta_{1}}(t)$ take $v=$ $u_{\eta_{2}}(t)$ and also in the same inequality satisfied by $u_{\eta_{2}}(t)$ take $v=u_{\eta_{1}}(t)$. Using (3.2), (3.5) (b), (3.6) and (3.7) (b), we obtain after adding the resulting inequalities that

$$
\left\|T_{t}\left(\eta_{1}\right)-T_{t}\left(\eta_{2}\right)\right\|_{V} \leq \frac{\|\mu\|_{L^{\infty}\left(\Gamma_{3}\right)} L_{p} d_{\Omega}^{2}}{m}\left\|\eta_{1}-\eta_{2}\right\|_{V}, \forall \eta_{1}, \eta_{2} \in K
$$


Thus if $\left.\|\mu\|_{L^{\infty}\left(\Gamma_{3}\right)} L_{p} d_{\Omega}^{2}\right) / m<1$, then the map $T_{t}$ is a contraction; it has a unique fixed point $\eta^{*}$ and $u_{\beta \eta^{*}}(t)$ is a unique solution of the inequality (4.3). Next, denote $u_{\beta \eta^{*}}(t)=u_{\beta}(t)$ for each $t \in[0, T]$. As in [35], to show that $u_{\beta} \in C([0, T] ; K)$, it suffices to see from (4.3) that there exists a constant $c>0$ such that

$$
\begin{aligned}
& \left\|u_{\beta}\left(t_{1}\right)-u_{\beta}\left(t_{2}\right)\right\|_{V} \leq \\
& \frac{c}{m-\|\mu\|_{L^{\infty}\left(\Gamma_{3}\right)} L_{p} d_{\Omega}^{2}}\left(\left\|f\left(t_{1}\right)-f\left(t_{2}\right)\right\|_{V}+\left\|\beta\left(t_{1}\right)-\beta\left(t_{2}\right)\right\|_{L^{2}\left(\Gamma_{3}\right)}\right) \\
& \forall t_{1}, t_{2} \in[0, T] .
\end{aligned}
$$

Therefore, as $f \in C([0, T] ; V)$ and $\beta \in C\left([0, T] ; L^{2}\left(\Gamma_{3}\right)\right)$, we immediately conclude (4.4).

In the second step, we consider the following problem.

Problem $P_{2 \beta}$. Find $\chi_{\beta}:[0, T] \rightarrow L^{2}\left(\Gamma_{3}\right)$ such that

$$
\begin{gathered}
\dot{\chi}_{\beta}(t)=H_{a d}\left(\chi_{\beta}(t), R\left(\left|u_{\beta \tau}(t)\right|\right)\right) \text { a.e. } t \in(0, T), \\
\chi_{\beta}(0)=\beta_{0} .
\end{gathered}
$$

We obtain the following result.

Lemma 4.5. Problem $P_{2 \beta}$ has a unique solution $\chi_{\beta}$ which satisfies

$$
\chi_{\beta} \in W^{1, \infty}\left(0, T ; L^{2}\left(\Gamma_{3}\right)\right) \cap B .
$$

Proof. Consider the mapping $F_{\beta}(t, \theta):[0, T] \times L^{2}\left(\Gamma_{3}\right) \rightarrow L^{2}\left(\Gamma_{3}\right)$ defined by

$$
F_{\beta}(t, \theta)=H_{a d}\left(\theta, R\left(\left|u_{\beta \tau}(t)\right|\right)\right) .
$$

It follows from the properties of the truncation operator $R$, that $F_{\beta}$ is Lipschitz continuous with respect to the second argument, uniformly in time. Moreover, for any $\theta \in L^{2}\left(\Gamma_{3}\right)$, the mapping $t \rightarrow F_{\beta}(t, \theta)$ belongs to $L^{\infty}(0$, $\left.T ; L^{2}\left(\Gamma_{3}\right)\right)$. Then, from a version of Cauchy-Lipschitz theorem, we deduce the existence of a unique fonction $\chi_{\beta} \in W^{1, \infty}\left(0, T ; L^{2}\left(\Gamma_{3}\right)\right)$, which satisfies (4.7), (4.8). The regularity $\chi_{\beta} \in B$, follows from (4.7), (4.8) and (3.12), (see $[27,28,30])$. Therefore, from Lemma 3.5 , we deduce that for all $\beta \in X$, the solution $\chi_{\beta}$ of Problem $P_{2 \beta}$ belongs to $X$. Next, we define the mapping $\Lambda: X \rightarrow X$ by

$$
\Lambda \beta=\chi_{\beta}
$$

The third step consists of the following lemma.

Lemma 4.6. The mapping $\Lambda$ has a unique fixed point $\beta^{*}$. 
Proof. We have

$$
\Lambda \beta(t)=\beta_{0}+\int_{0}^{t} H_{a d}\left(\chi_{\beta}(s), R\left(\left|u_{\beta \tau}(s)\right|\right)\right) d s,
$$

where $u_{\beta}$ is the solution of Problem $P_{1 \beta}$. Then for $\beta_{1}, \beta_{2} \in X$, using (3.10) (a) and the properties of $R$ see ([27]), we get

$$
\begin{aligned}
& \left|\chi_{\beta_{1}}(t)-\chi_{\beta_{2}}(t)\right| \leq \\
& L_{a d} \int_{0}^{t}\left|\chi_{\beta_{1}}(s)-\chi_{\beta_{2}}(s)\right| d s+L_{a d} \int_{0}^{t}\left|u_{\beta_{1} \tau}(s)-u_{\beta_{2} \tau}(s)\right| d s .
\end{aligned}
$$

Applying Gronwall's inequality and using (3.2), it follows that there exists a constant $c_{1}>0$ such that

$$
\left\|\chi_{\beta_{1}}(t)-\chi_{\beta_{2}}(t)\right\|_{L^{2}\left(\Gamma_{3}\right)} \leq c_{1} \int_{0}^{t}\left\|u_{\beta_{1}}(s)-u_{\beta_{2}}(s)\right\|_{V} d s
$$

Let now $t \in[0, T]$. Then, using the inequality (4.3), (3.5), (3.6), (3.7), (3.8) and (3.9), we deduce for $\|\mu\|_{L^{\infty}\left(\Gamma_{3}\right)}<m / L_{p} d_{\Omega}^{2}$ that (see [35])

$$
\left\|u_{\beta_{1}}(t)-u_{\beta_{2}}(t)\right\|_{V} \leq c_{2}\left\|\beta_{1}(t)-\beta_{2}(t)\right\|_{L^{2}\left(\Gamma_{3}\right)}
$$

for some constant $c_{2}>0$. Hence, it follows that there exists a constant $c_{3}>0$ such that

$$
\left\|\Lambda \beta_{1}(t)-\Lambda \beta_{2}(t)\right\|_{L^{2}\left(\Gamma_{3}\right)} \leq c_{3} \int_{0}^{t}\left\|\beta_{1}(s)-\beta_{2}(s)\right\|_{L^{2}\left(\Gamma_{3}\right)} d s \quad \forall t \in[0, T] .
$$

Therefore, we obtain

$$
\left\|\Lambda \beta_{1}-\Lambda \beta_{2}\right\|_{X} \leq \frac{c_{3}}{k}\left\|\beta_{1}-\beta_{2}\right\|_{X}, \forall \beta_{1}, \beta_{2} \in X
$$

Thus, this previous inequality shows that for $k>c_{3}, \Lambda$ is a contraction. Then it has a unique fixed point $\beta^{*}$ which satisfies (4.7) and (4.8). On the other hand from (4.6) we deduce that $u_{\beta^{*}} \in W^{1, \infty}(0, T ; V)$.

Proof of Theorem 4.1. Let $\beta=\beta^{*}$ and let $u_{\beta^{*}}$ the solution to Problem $P_{1 \beta}$. We conclude by $(4.3),(4.7)$ and $(4.8)$ that $\left(u_{\beta^{*}}, \beta^{*}\right)$ is a solution of Problem $P_{2}$. Now to prove the uniqueness of the solution, suppose that $(u, \beta)$ is a solution of Problem $P_{2}$ which satisfies (3.12), (3.13) and (3.14). It follows from (3.12) that $u$ is a solution of Problem $P_{1 \beta}$ and by Proposition 3.2 we get $u=u_{\beta}$. Taking $u=u_{\beta}$ in (3.13) and using the initial condition (3.14), we deduce that $\beta$ is a solution of Problem $P_{2 \beta}$. Finally, using Lemma 3.5, we obtain $\beta=\beta^{*}$ and then $\left(u_{\beta^{*}}, \beta^{*}\right)$ is a unique solution to Problem $P_{2}$ which satisfies (4.1), (4.2). 
Remark 4.7. The question concerning the problem with a great coefficient of friction is not resolved here and remains still open.

Aknowledgement. The authors wish to thank the anonymous referee to his remarks and suggestions.

\section{References}

[1] L.-E. Andersson, Existence result for quasistatic contact problem with Coulomb friction, Appl. Math. Optimiz., 42, (2000), 169-202

[2] H. T. Banks, S. Hu, and Z. R Kenz, A brief review of elasticity and viscoelasticity for solids, Adv. Appl. Math. Mech., 3, (2011), 1-51

[3] L. Cangémi, Frottement et adhérence: modèle, traitement numérique et application à l'interface fibre/matrice, Ph.D. Thesis, Univ. Méditerranée, Aix Marseille I, 1997

[4] O. Chau, J. R. Fernandez, M. Shillor, and M. Sofonea, Variational and numerical analysis of a quasistatic viscoelastic contact problem with adhesion, Journal of Computational and Applied Mathematics, 159, (2003), 431-465

[5] O. Chau, M. Shillor, and M. Sofonea, J. Appl. Math. Phys. (ZAMP), 55, (2004), $32-47$

[6] M.Cocou, E. Pratt, and M. Raous, Formulation and approximation of quasistatic frictional contact, Int.J.Engng Sc., 34, (1996), 783-798

[7] M. Cocou and R. Rocca, Existence results for unilateral quasistatic contact problems with friction and adhesion, 34, (2000), 981-1001

[8] M. Cocou, M. Schyvre, and M. Raous, A dynamic unilateral contact problem with adhesion and friction in viscoelasticity, Z. Angew. Math. Phys., 61, (2010), 721743

[9] S. Drabla and Z. Zellagui, Analysis of a electro-elastic contact problem with friction and adhesion, Studia Univ. "Babes-Bolyai", Mathematica, LIV, (2009)

[10] G. Duvaut, C. R. Acad. Sc. Paris Série A, 290, (1980), 263

[11] G. Duvaut and J-L Lions, Les inéquations en mécanique et en physique, Dunod, Paris, 1972

[12] C. Eck, J. Jarušek, and M. Krbec, Unilateral Contact Problems. Variational Methods and Existence Theorems, Chapman \& Hall / CRC (Taylor \& Francis Group), Boca Raton-London-New York-Singapore, 2005

[13] J. R. Fernandez, M. Shillor, and M. Sofonea, Analysis and numerical simulations of a dynamic contact problem with adhesion, 37, (2003), 1317-1333

[14] M. Frémond, Adhérence des solides, J. Mécanique Théorique et Appliquée, 6, (1987), 383-407

[15] M. Frémond, Equilibre des structures qui adhèrent à leur support, C. R. Acad. Sci. Paris, 295, (1982), 913-916 
[16] M. Frémond, Non smooth Thermomechanics, Springer, Berlin, 2002

[17] J. Jarusěk and M. Sofonea, On the solvability of dynamic elastic-visco-plastic contact problems, Zeitschrift fur Angewandte Mathematik and Mechanik, (ZAMM), 88, (2008), 3-22

[18] J. Jarusěk and M. Sofonea, On the of dynamic elastic-visco-plastic contact problems with adhesion, Annals of AOSR, Series on Mathematics and its applications, $\mathbf{1}$ (2009), 191-214

[19] S. Migorski, A. Ochal, and M. Sofonea, Nonlinear Inclusions and Hemivariational Inequalities, Models and Analysis of Contact Problems, Advances in Mechanics and Mathematics, 26, (2013)

[20] M. Migorski, A. Ochal, and M. Sofonea, Analysis of a quasistatic contact problem for piezoelectric materials, J. Math. Anal. Appl., 382, (2011), 701-713

[21] S. Migorski, A. Ochal, and M. Sofonea, A dynamic frictional contact problem for piezoelectric materials, J. Math. Anal. Appl., 361, (2010), 161-176

[22] S.A. Nassar, T. Andrews, S. Kruk, and M. Shillor, Modelling and Simulations of a bonded rod, Math. Comput. Modelling, 42, (2005), 553-572

[23] M. Raous, L. Cangémi, and M. Cocu, A consistent model coupling adhesion, friction, and unilateral contact, Comput.Meth. Appl. Mech. Engng., 177, (1999), 383399

[24] R. Rocca, Analyse et numérique de problèmes quasi-statiques de contact avec frottement local de Coulomb en élasticité, Thèse, Aix Marseille 1, 2005

[25] J. Rojek and J. J. Telega, Contact problems with friction, adhesion and wear in orthopeadic biomechanics. I: General developements, J. Theor. Appl. Mech., 39, (2001), 655-677

[26] M. Shillor, M. Sofonea, and J. J. Telega, Models and Variational Analysis of Quasistatic Contact, Lecture Notes Physics, 655, (2004)

[27] M. Sofonea, W. Han, and M. Shillor, Analysis and Approximation of Contact Problems with Adhesion or Damage, Pure and Applied Mathematics, 276, (2006)

[28] M. Sofonea and T.V. Hoarau -Mantel, Elastic frictionless contact problems with adhesion, Adv. Math. Sci. Appl, 15, (2005), 49-68

[29] M. Sofonea and A. Matei, Variational inequalities with applications, Advances in Mathematics and Mechanics, 18, (2009)

[30] M. Sofonea and A. Matei, An elastic contact problem with adhesion and normal compliance, Journal of Applied Analysis, 12, (2006), 19-36

[31] M. Sofonea, F. Patrelescu, and A. Farcas, A viscoplastic contact problem with normal compliance, unilateral constraint and memory term, Applied Mathematics 8 Optimization, 69, (2014), 175-198

[32] M. Sofonea and F. Patrelescu, Analysis of a history-dependent frictionless contact problem, Mathematics and Mechanics of solids, 18, (2012), 409-430

[33] M. Sofonea and A. Matei, Mathematical models in Contact Mechanics, London, Mathematical Society, Lecture Notes, Cambridge University, Press 398, Cambridge, 2012 
[34] A. Touzaline, Frictionless contact problem with finite penetration for elastic materials, Ann. Pol. Math., 98, (2010), 23-38

[35] A. Touzaline, Analysis of a contact adhesive problem with normal compliance, Ann. Pol. Math., 104, (2012), 175-188

Arezki Touzaline

Laboratoire de Systèmes Dynamiques

Faculté de Mathématiques, USTHB

BP 32 EL ALIA

Bab-Ezzouar, 16111, Algérie

E-mail: ttouzaline@yahoo.fr

Rachid Guettaf

Faculté des Sciences Campus Sud,

UMBB-35000 Boumerdès, Algérie

E-mail: r_guettaf@yahoo.fr

Received: 12.04.2014

Accepted: 26.07.2014

Revised: 18.07.2014 